\title{
Conrady's Chromatic Condition
}

\author{
Donald P. Feder
}

\begin{abstract}
Nearly fifty years ago Conrady published a simple and ingenious formula for determining the state of chromatic correction of an optical system. This method, called the " $D$ - $d$ achromatism", depends upon the variation of the optical path as a function of wavelength. It can be used to evaluate both axial and lateral color, chromatic variation of spherical aberration, and secondary chromatic aberration. Unfortunately, this method is not as well known or as widely used as it deserves to be.

This paper proves the exact equation and discusses an extension of it which is approximate. A numerical example illustrates the accuracy obtainable with the approximation. The principal applications are presented and formulas derived giving the relations between the "D- $d$ method" and conventional means of expressing the chromatic aberration.
\end{abstract}

\section{Introduction}

Professor Conrady is among the best-known teachers of the art of optical design. His book "Applied optics and optical design" is familiar to most designers and is a bible to some. The "D-d method" for calculating chromatic aberration, however, is not found in his book, and this may account for the fact that it is not as widely known as it deserves to be. The method was published in two papers in the Monthly Notices of the Royal Astronomical Society nearly fifty years ago. ${ }^{2}$ It was surprising to find recently that many good lens designers are unacquainted with the method. Furthermore, even those who use it do not often apply it to off-axis imagery.

It will be shown in this paper, that after rays have been traced through the system to determine the monochromatic aberrations, the complete state of the chromatic correction can then be found with very little extra work and without the necessity of tracing rays at any other wavelength. In addition the $D-d$. method gives the results of ray tracing at three wavelengths. Because this method gives answers in the form of optical path differences, it is frequently easier to obtain a well corrected system by this method than by conventional methods. The use of the $D$-d method is recommended to designers who require a simple and elegant way for finding the chromatic aberration of an optical system over the entire field.

The proof presented by Conrady is not entirely satisfactory, and later proofs, although more cumbersome, are less convincing. Some of them depend upon very complicated geometrical figures that are difficult to follow.

The region of validity of the formula is not well defined. A recent paper, for example, stated that the chromatic variation of spherical aberration is not always given correctly by the formula. Conrady, himself, appeared to think that the secondary chro-

${ }_{1}^{1}$ This work was done as part of a research project sponsored by the U. S. A ir Force.

2 A. E. Conrady, Monthly Notices of the Royal Astronomical Society, p. 182 (Jan. 1904); p. 458 (March 1904). matic aberration ${ }^{3}$ was exactly determined by the $D-d$ method. It will be seen below, however, that any aberration determined by the first derivative with respect to wavelength is precisely given by this formula. Therefore, any chromatic aberration, including chromatic variation of spherical, is accurately given over a narrow wavelength region in the neighborhood of the median wavelength no matter what the monochromatic aberrations of the system may be. For a somewhat wider wavelength region the method gives an approximate answer, which is ordinarily good enough to determine the secondary chromatic aberration of the system.

\section{Derivation of Principal Equation}

Figure 1 shows a meridan section through a typical lens. The proof will be given for this case and immediately extended to any number of elements. A point source at $O$ is emitting light that is refracted by the lens. For a particular wavelength $\lambda_{0}$, we consider a wave front in the image space. We may suppose that this wave front is located a considerable distance from the image of $O$. We take a point, $P$, lying on the wave front, and draw the normal to the wave front. This normal coincides with a ray in the image space whose extension in the object space passes through $O$. This ray is shown as a solid line in figure 1 . In like manner for any wavelength $\lambda$, there exists a unique ray passing from $O$ to $P$. Another such ray is shown by the dotted line in figure 1.

In order to find the chromatic aberration, we should like to know what happens to the wave front when the wavelength is varied. We first consider what happens at the point $P$, and then, by letting $P$ move over the wave front, obtain the total effect. We first mark off along the ray the distances $D_{1}$, $D_{2}, D_{3}, D_{4}$ intercepted on the ray by the various media. The distance $D_{4}$ is the distance along the ray from the last surface to the point $P$. As the wave-

${ }^{3}$ Because of strong objections by some people to the term secondary spectrum the expression secondary chromatic aberration is used for this quantity throughou the paper. 


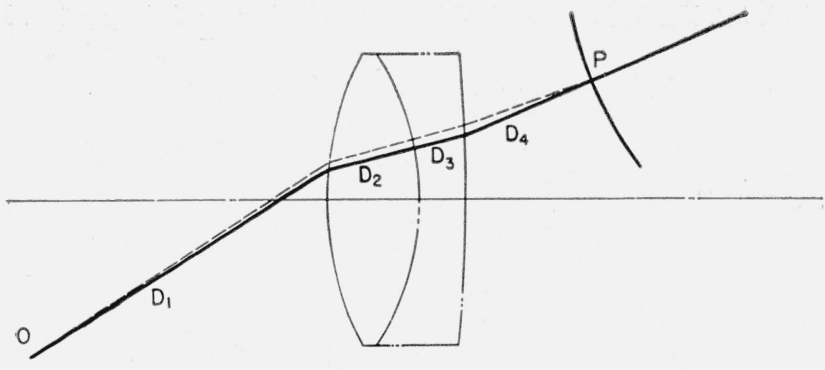

Figure 1. Meridian section of typical lens.

Light from a point source at $O$ traverses a typical lens producing an emergent wave front at $P$ in light of wavelength $\lambda_{0}$. The solid line shows such a ray with the distances in the various media indicated by $D_{1}, D_{2}, D_{3}, D_{4}$. The dotted line indicates a ray going from $O$ to $P$ for another wavelength $\lambda$. The wave fron for $\lambda$ is not shown in the figure.

length changes, the distances $D_{1}, D_{2}, D_{3}, D_{4}$, change and so are to be considered functions of $\lambda$.

Let us define $V(\lambda)$ to be the optical-path length from $O$ to $P$. Suppose, for convenience, that the object and image are in air; then

$$
V(\lambda)=D_{1}+N_{2} D_{2}+N_{3} D_{3}+D_{4},
$$

where $N_{1}$ and $N_{2}$ are the refractive indices of the corresponding media. Differentiating $V$ with respect to $\lambda$ gives the rate of change of the optical-path length from $O$ to $P$. One obtains

$$
\begin{aligned}
\frac{d V}{d \lambda}=\left\{\frac{d D_{1}}{d \lambda}+N_{2} \frac{d D_{2}}{d \lambda}+N_{3} \frac{d D_{3}}{d \lambda}\right. & \left.+\frac{d D_{4}}{d \lambda}\right\} \\
& +\left\{\frac{d N_{2}}{d \lambda} D_{2}+\frac{d N_{3}}{d \lambda} D_{3}\right\}
\end{aligned}
$$

One sees that the derivative is divided naturally into two parts. In the first part (left-hand bracket), the refractive indices are held fixed. Therefore, this part results from the variation of the path of the ray and is zero by Fermat's principle.

Fermat's principle states that the optical-path length between two fixed points possesses a stationary value with respect to arbitrary variations of the mechanical path. In this case we are considering a subset of all the possible variations in path from $O$ to $P$ : namely, only such variations as are generated by varying $\lambda$. Because of the manner in which this subset was chosen, it is clear that it contains, for light of any fixed wavelength $\lambda$, the actual path traversed by the light. Furthermore, for light of this wavelength $\lambda$, the actual path possesses a stationary value. In mathematical language,

$$
d D_{1} / d \lambda+N_{2} d D_{2} / d \lambda+N_{3} d D_{3} / d \lambda+d D_{4} / d \lambda=0 .
$$

It follows that $d V / d \lambda=D_{2} d N_{2} / d \lambda+D_{3} d N_{3} / d \lambda$. This equation can be immediately generalized to any number of elements giving

$$
\frac{d V}{d \lambda}=\sum \frac{d N}{d \lambda} D
$$

This is the principal equation, and forms the basis for the method. It should be noted that nothing in its derivation restricts its application to axial object points or even to meridian rays.

\subsection{Significance of Principal Equation}

To understand the significance of eq (1) recall that for light of a fixed wavelength, the optical-path length, $V$, from the object point to any point on the wave front is a constant. Suppose that $d V / d \lambda$ has been calculated for all points $P$ on the wave front of wavelength $\lambda$. For a sufficiently small increment in wavelength, $\Delta \lambda$, it is approximately true that $\Delta V=(d V / d \lambda) \Delta \lambda$. A point $P^{\prime}$ is now defined such that $P^{\prime}$ lies on the ray through $P$, and such that the displacement of $P^{\prime}$ from $P$ is $-\Delta V$. It is clear that the optical-path length $V^{\prime}$ from $O$ to $P^{\prime}$ for light of wavelength $\lambda+\Delta \lambda$ is equal to $V$. In this manner a set of points $P^{\prime}$ is constructed, which constitutes a wave front for light of wavelength $\lambda+\Delta \lambda$. By the nature of the construction, the displacement of this wave front from the original wave front is $-(d V / d \lambda) \Delta \lambda$. This equation becomes exact only in the limit as $\Delta \lambda \rightarrow 0$.

The above argument shows that if $\Delta V$ is a constant over the entire wave front, then the changed wave front is "parallel" to the original one, and there is no chromatic aberration in the image of $O$. Stated more precisely, if $d V / d \lambda$, evaluated at $\lambda=\lambda_{0}$, is the same at all points on the wave front, there is no chromatic aberration in the wavelength region around $\lambda_{0}$. On the other hand, the variation of $d V / d \lambda$, as $P$ is moved over the wave front, is a measure of the chromatic aberration. A more detailed study of this point is given later in this paper.

The actual calculation of $d V / d \lambda$ from eq (1) is a simple matter. After the monochromatic errors are determined by tracing rays at a central wavelength, the values of the $D^{\prime}$ s are available as a byproduct. The quantities $d N / d \lambda$ are available from the known properties of the glasses employed. It is a simple matter to form the indicated sum for several points on the wave front and to examine the chromatic aberration in the vicinity of the central wavelength

\subsection{The Approximate Equation}

Now one may ask how to determine the secondary chromatic aberration. For this it is necessary to calculate $d^{2} V / d \lambda^{2}$. As eq (1) holds for all values of $\lambda$, it may be differentiated, yielding

$$
d^{2} V / d \lambda^{2}=\Sigma D d^{2} V / d \lambda^{2}+\Sigma(d N / d \lambda)(d D / d \lambda) .
$$

The first term of eq (2) is easy to obtain, but the second term is more troublesome. Unfortunately, it is not zero, but computations on several widely different systems show that it must be considerably smaller than the term $\Sigma D d^{2} N / d \lambda^{2}$. If this term, and all terms of higher order in $\Delta \lambda$, are neglected, then one has

$$
\Delta V=\frac{d V}{d \lambda} \Delta \lambda+\frac{d^{2} V}{d \lambda^{2}} \frac{(\Delta \lambda)^{2}}{2}=\Sigma D\left\{\frac{d N}{d \lambda} \Delta \lambda+\frac{d^{2} N}{d \lambda^{2}} \frac{(\Delta \lambda)^{2}}{2}\right\},
$$


or finally,

$$
\Delta V=\Sigma D \Delta N .
$$

Equation (3) is an approximate equation, but it gives more information than eq (1) because it takes partial account of all higher derivatives of $N$ with respect to $\lambda$. Furthermore, it is much easier to apply than eq (1) because the glass catalogs list the indices for discrete wavelengths, and in many cases list the values of $\Delta N$ directly. On the other hand, in order to find $d N / d \lambda$ for use in eq (1) it is necessary to fit one of the various dispersion formulas to the glass data and to compute $d N / d \lambda$ for the wavelength at which the rays were traced. Although this is a straightforward procedure, and has the advantage of being mathematically exact, the use of eq (3) not only entails less work but also gives an approximate idea of the secondary chromatic aberration.

\subsection{Modification When Final Medium Is Not Air}

In the case that the final image is not formed in air, then $-\Delta V / N^{\prime}$ rather than $-\Delta V$ is the correct expression for the displacement of the wave front. Here $N^{\prime}$ is the index of the final medium.

It must also be remembered that the sum $\Sigma D \Delta N$ should include the term $D^{\prime} \Delta N^{\prime}$ for the last medium, where $D^{\prime}$ is the distance along the ray from the last refracting surface to the focal surface.

\section{Application of Method}

After rays have been traced through the system to determine the monochromatic errors, it is necessary to determine the $D$ 's in each medium for which $\Delta N \neq 0$. Fortunately, in the usual lens there are several air spaces (where $\Delta N=0$ ), and these disappear from the summation in eq (3). In some ray-tracing schemes the values of the $D$ 's are immediately available for each medium, and in others these quantities are not directly given. Even in the latter event, however, they can easily be computed from the ray-tracing data. A common method for meridian rays employs the angle of incidence, $I$, and the angle of inclination, $U$, as coordinates of the ray. In this case one has

$$
D=r_{1} \cos I_{1}^{\prime}-r_{2} \cos I_{2}+\left(t_{12}-r_{1}+r_{2}\right) \cos U_{12},
$$

where $I_{1}^{\prime}$ is the angle of refraction and $r_{1}$ the radius of the surface preceding the medium; $I_{2}$ is the angle of incidence, and $r_{2}$ is the radius of the surface following the medium, and $U_{12}$ is the angle of inclination of the ray in the medium of thickness $t_{12}$.

The values of the $D$ 's need not be obtained with high precision because each $D$ is multiplied by a small number, which is rarely larger than 0.03. This is an important point from the standpoint of the practical computer. Five place accuracy in the $D$ 's is more than sufficient for even the most precise work.

After the values of the $D$ 's have been computed by any suitable method, one forms for each traced ray the sum $\Sigma D \Delta N=\Delta V$. If one is achromatizing for the region from $F$ to $C$, then $N_{F}-N_{C}$ for $\Delta N$ is used. Then $\Delta V$ is plotted against some variable that is a measure of the position of the ray in the aperture. For meridian rays this variable may be the coordinate of the ray in the entrance pupil, the tangent of the slope angle of the image ray, or any variable that locates the ray in the aperture. For reasons of convenience, which will appear later, we shall use in this paper $\sin U$, where $U$ is the angle of inclination of the image ray.

\subsection{Interpretation}

\section{a. Axial Object Points}

If, for the axial bundle of rays, one plots the value of $\Delta V$ against $\sin U$, a curve that is symmetrical about the line $\sin U=0$ is obtained. The displacement of the curve is immaterial, and it is customary to subtract from each ordinate the value of $\Delta V$ at the origin. This value is $\Sigma d \Delta N$, where $d$ is the axial distance between surfaces. One obtains $\Sigma(D-d) \Delta N$, and this is plotted against $\sin U$. This is the formula that gives rise to the popular name for the method, the $D-d$ method.

If the lens has no longitudinal chromatic aberration, the curve will be a straight line (see fig. 2). If simple primary aberration is present, the curve will be approximately parabolic and will be concave upward in the case of overcorrection and concave downward for undercorrection. (In establishing a sign convention, $\Delta N$ is taken to be positive for all the media, and $\sin U$ is taken to be positive when the final ray slopes downward to the right. It should be noted that if $\Delta V$ is positive, the wave front for $\lambda+\Delta \lambda$ is behind the wave front for $\lambda$.) A simple relation between the curvature of the parabola and the longitudinal aberration is derived in the final section.

Usually the situation is complicated by the presence of chromatic variation of spherical aberration. In many lenses initial undercorrection is followed by marginal overcorrection producing a zone. A case of this type is shown in figure 2. Good correc-

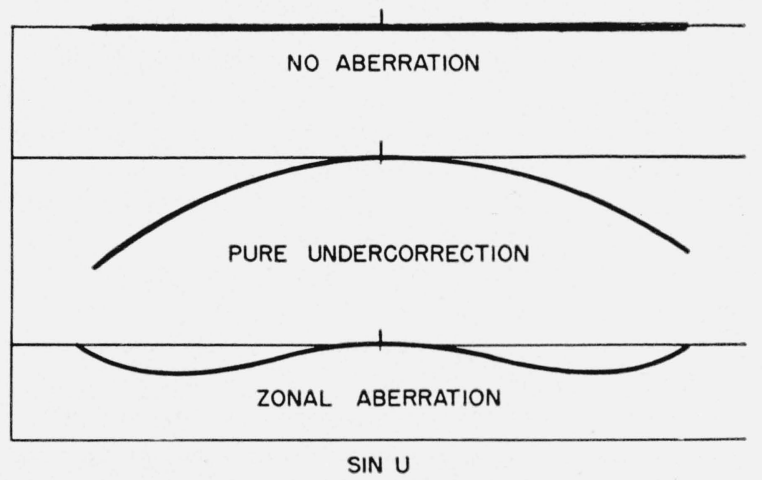

Figure 2. Axial fan.

The ordinate for each curve is $\Sigma(D-d) \Delta N$. As these curves are for axial points, they are necessarily symmetrical about the line $\sin U=0$. 


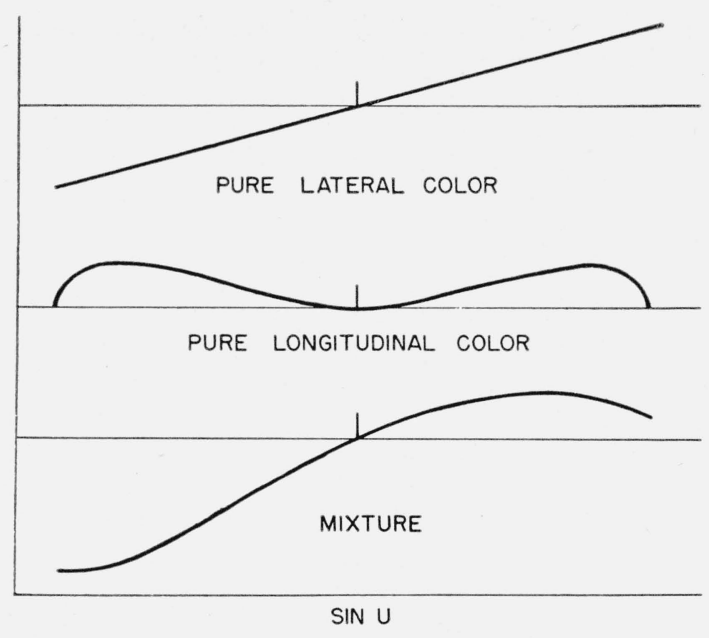

Figure 3. Oblique fan.

The ordinate for each curve is $\Delta V$. Because the displacement is of no impor tance, each curve can be zeroed about the chief ray. If the curve shows symmetry about the value of sin $U$ corresponding to the chief ray, then only pure longitudinal color is present. A tilt indicates lateral color.

tion here makes $\Sigma(D-d) \Delta N=0$ at the margin. Then wave fronts that are tangent in the center of the aperture will cross at the margin. At a point usually near the 0.7 zone the wave fronts will be parallel and the corresponding rays coincident.

\section{b. Extra-Axial Object Points}

As mentioned above, for meridian rays one plots $\Delta V$ against $\sin U$, where $U$ is the slope angle of the final ray in the image space and $\Delta V=\Sigma D \Delta N$. In this case, the symmetry present on axis is no longer inherent. Figure 3 depicts some of the characteristic types of chromatic aberration that may be present in oblique bundles. If the curve is a straight line inclined to the $\sin U$ axis, then pure lateral aberration is present. One may think of the wave fronts as being inclined to one another. The lateral aberration is proportional to the slope of the line, and its numerical value can readily be obtained (see section 3.3). As before the displacement of the curve is immaterial, only the differences in $\Delta V$ being significant. If the curve is symmetrical about a line parallel to the $\Delta V$ axis through the chief ray, then pure longitudinal aberration is present. The longitudinal aberration for an off-axis object point may be quite different from that present on the axis.

In the ordinary case the curve possesses neither symmetry nor straightness, and the aberration is a mixture of lateral and longitudinal chromatic aberration.

In the final stages of an optical design, one may have curves of $\Delta V$ against $\sin U$ for several different object points. It is desirable to obtain the best correction throughout the usable field. An advantage of this method is that the chromatic aberration is represented in the same manner both on and off axis.

The skew-ray aberration is also represented by $\Delta \mathrm{V}$, and in some cases it may be desirable to at least plot the sagittal rays. The author believes, however, that the chromatic aberration of the skew rays will not be troublesome if the meridian rays are properly corrected.

\section{c. Secondary chromatic aberration}

To obtain an estimate of the secondary chromatic aberration, one should recompute $\Delta V$ by using a different set of $\Delta N$. Suppose, for example, that the central wavelength is sodium $D$ and that rays have been traced in this color to determine the monochromatic aberrations. It is desired to correct the lens in the spectral region $C$ to $F$. The procedure might be as follows:

1. The sum $\Sigma(D-d) \Delta N$ is made zero at the margin using $N_{F}-N_{C}$ for $\Delta N$ and using the values of the $D$ 's obtained from ray tracing in sodium light.

2 . Check $\Sigma(D-d) \Delta N$ to make sure that it is satisfactory over the whole aperture. There is no point in worrying about the secondary chromatic aberration if there is a large amount of chromatic variation of spherical aberration present.

3. After $\Sigma(D-d) \Delta N$ is satisfactory over the aperture, it can be recomputed by using the value $N_{F}-N_{D}$ for $\Delta N$ and the same values for the $D$ 's as before. The value of this second sum is an indication of the secondary chromatic aberration. There will be some error owing to the fact that eq (3) involves an approximation, but this is not serious for most purposes.

It should be recognized that the important use of the D-d method is to calculate the higher order chromatic aberrations. Before applying this method, a competent designer will already know the primary values of the longitudinal and lateral color and will have reduced these aberrations to reasonably small values.

If the secondary chromatic aberration is to be corrected, it must first be approximately corrected in the paraxial region. The simplest way to determine this is by tracing paraxial rays for the separate wavelengths. After paraxial correction is made, it is necessary to apply the $D$ - $d$ method as listed in steps 1,2 , and 3 above to ascertain whether good correction has been achieved over the entire aperture.

\subsection{Tolerances}

One can set up a tolerance for the variation of $\Delta V$ on the basis of physical optics. Suppose that the image in $D$ light is sharp and that $\Delta V$ calculated from $F$ to $D$ does not vary more than one-fourth wave over the aperture. Then at the $D$ focus the image in $F$ light will be sensibly perfect within the meaning of the Rayleigh limit. Similarly, if the variation in $\Delta V$ calculated from $D$ to $C$ is also less than one-fourth wave, then the image in $C$ light observed at the $D$ focus will be sharp. Under such circumstances the chromatic aberration would be extremely well corrected over the entire region from $F$ to $C$. On the other hand, the above tolerance is extremely strict and will seldom be met in practice. Usually one corrects the axial bundle of rays so that $\Sigma(D-d) \Delta N$ 
is zero at the edge of the aperture, and this correction is ordinarily quite adequate. If there is a large zone because of the presence of chromatic variation of spherical aberration, then a basic change in the design is necessary to reduce this aberration. If the secondary chromatic aberration is to be corrected, provision for this correction must be made in the preliminary design.

In lenses covering an appreciable field, the curves for several obliquities must be simultaneously corrected, and this may lead to sacrificing best axial correction in order to gain at the edge of the field. The $D$-d method is ideally suited to making such decisions because the chromatic aberration from axial and extra-axial object points are both represented by the same type of graph.

In very many cases the tolerances are determined by a combination of experience and such overriding considerations as cost and complexity.

\subsection{Relation Between Ray and Wave-Front Aberrations}

For designers who are more familiar with rayintercept methods for expressing chromatic aberration, it should be helpful to have a formula relating one to the other. Such a formula is derived below for meridian rays.

One considers a wave front in light of wavelength $\lambda$ located a distance $R$ from the focal plane (see fig. 4 ). When the wavelength is changed by $\Delta \lambda$, the changed wave front makes an angle $\delta$ with the original wave front at some arbitrary point $P$. It is clear that $d(\Delta V) / d s=\delta$, where $s$ is the arc length measured along the wave front. Also $s=R U$ because the wave front is nearly spherical. (Here $U$ is the

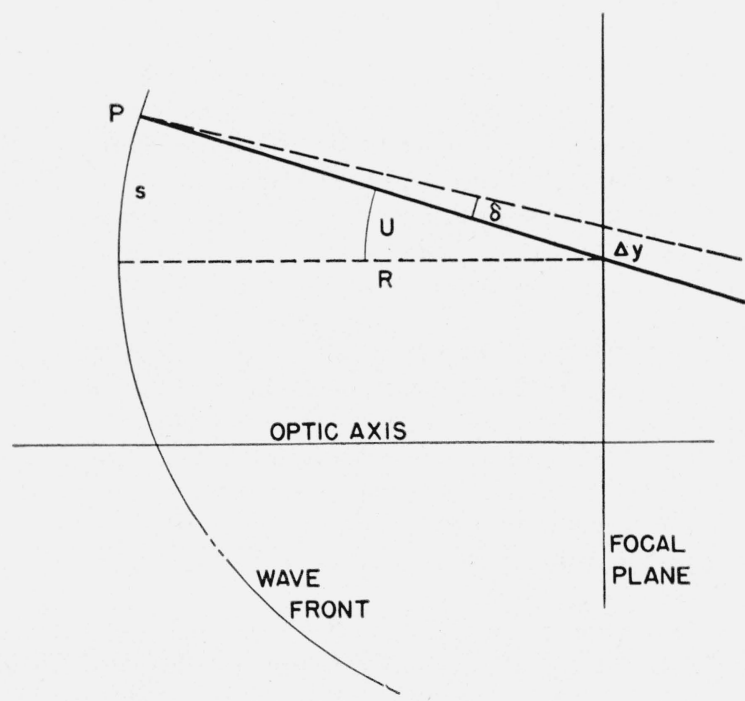

Figure 4.

The full curve shows a wave front in light of wavelength $\lambda$ located a distance $R$ from the focus. A ray normal to this wave front at $P$ makes an angle $U$ with the optic axis. A ray in light of wavelength $\lambda+\Delta \lambda$ makes an angle $\delta$ with the first ray. It is indicated by a dotted line through $P$ crossing the focal plane at a distance $\Delta y$ from the first ray. angle of inclination that the final ray makes with the optical axis.) Now one has

$$
\Delta y=\frac{R \delta}{\cos U}=\frac{R}{\cos U}\left\{\frac{d(\Delta V)}{d s}\right\} \text { and } d s=R d U .
$$

Then

$$
\Delta y=\frac{R}{\cos U}\left\{\frac{d(\Delta V)}{R d U}\right\}=\frac{1}{\cos U} \frac{d(\Delta V)}{d U} .
$$

Finally,

$$
\Delta y=\frac{d(\Delta V)}{d(\sin U)}
$$

The existence of eq (4) is the reason for plotting $\Delta V$ against $\sin U$ rather than against some other aperture variable, such as $\tan U$.

Equation (4) gives the displacement of the ray because of a change in $\lambda$. One can plot $\Delta V$ against $\sin U$ for several traced rays. By measuring the slope of the curve at any point, one finds the displacement between rays in the two wavelengths at that point. Using the formula, one can obtain the ray-intercept curve for any other wavelength after the curve has been found in the central wavelength. In practice, however, it is not usually desirable to do this because the single curve of $\Delta V$ against $\sin U$ is easier to interpret than two ray-intercept curves for different wavelengths.

\subsection{Numerical Example}

Figure 5,a, shows a curve obtained in an actual lens design. It represents the monochromatic aber-
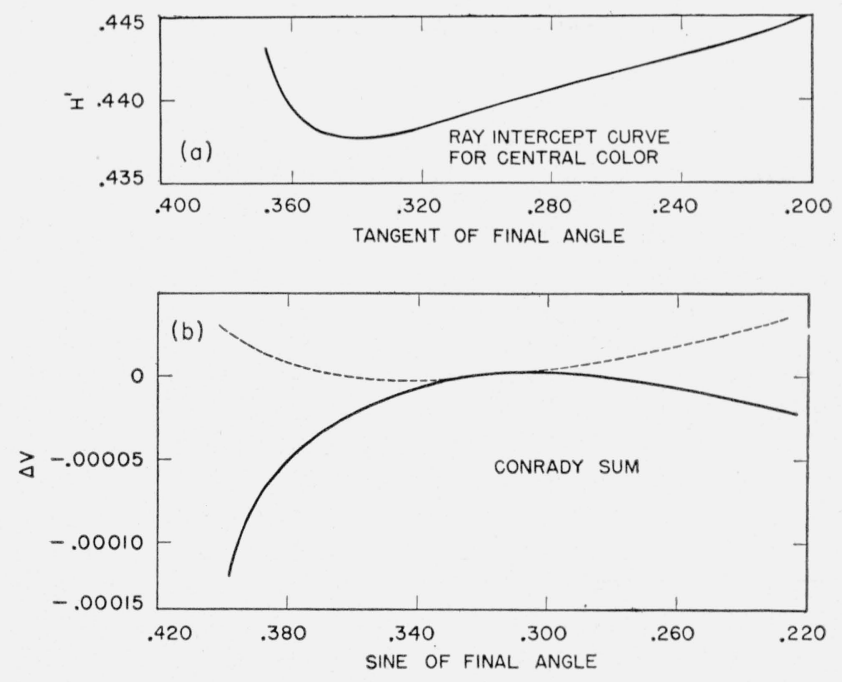

Figure 5 .

(a) The upper curve shows the meridian ray aberration at $60^{\circ}$ obliquity from the axis in $D$ light. The ordinate $H^{\prime}$ is the height of the ray on the focal plane, the abscissa is the tangent of the final angle. (b) The lower curves show $\Delta V$ for the same lens at the same obliquity. The solid curve is for the wave length region $F$ to $D$; the dotted curve from $C$ to $D$.

These curves show the ray intercept curves at $60^{\circ}$ in $F$ light (solid line) and
The $D$; the dotted curve from $C$ to $D$. $C$ light (dotted line) predicted by equation (4) from the curves in figure 5 . The crosses show the actual results of ray tracing in $\mathrm{F}$ light (solid curve) and the circles the results of ray tracing in $C$ light (dotted curve). 


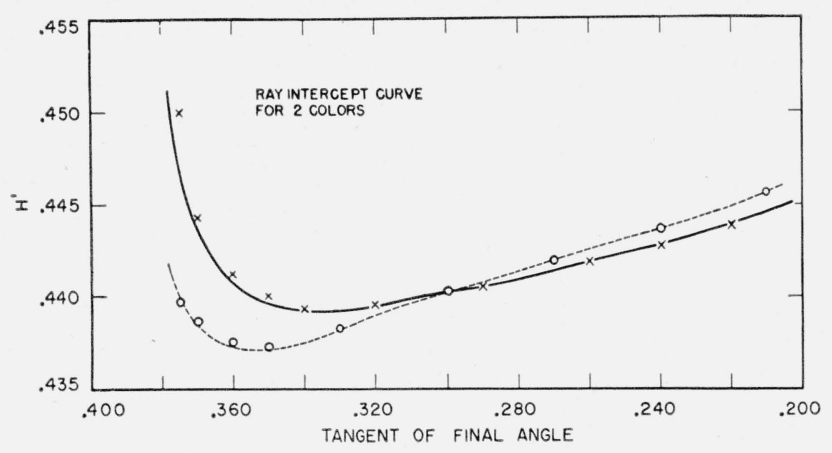

Figure 6. Ray-intercept curve for two colors.

ration for $D$ light for an object point $60^{\circ}$ from the optic axis. This lens was a complex system containing aspheric surfaces and was in an early stage of design. Figure 5,b, shows the curve of $\Delta V$ from $F$ to $D$ and the curve of $\Delta V$ from $C$ to $D$. Normally one would plot only the difference (that is, $\Delta V$ from $F$ to $C$ ), but in this case the ray-intercept curves for all three wavelengths were desired. From the slope of these curves, the values of $\Delta y$ have been calculated by eq (4). These differences were then added to the curve in figure 5,a, to obtain the curves in figure 6 and these curves checked by ray tracing. The crosses and circles show the position of the traced rays. The agreement is good except on the left end, where the aberration is large. The discrepancy is due to the fact that the wavelength interval used is not sufficiently small.

\subsection{Paraxial Correspondence}

One might ask the question how the curve for $\Sigma(D-d) \Delta N$ against aperture is related to the paraxial chromatic aberration. It would be possible to find this correspondence by an investigation of the limiting behavior of $\Sigma(D-d) \Delta N$ as the aperture is reduced to zero. Such an investigation, however, could only produce a well known formula giving the value of the longitudinal aberration in terms of the paraxial ray tracing data. This formula is reproduced below for reference.

Instead of deriving this formula from an optical path difference standpoint however, we shall attack the problem in a different manner, that is, by assuming that the longitudinal aberration is known and determining $\Sigma(D-d) \Delta N$ by using this information. Suppose there is a longitudinal difference in focus $\delta l$ between the image positions for two wavelengths. In the neighborhood of the axis each wave front is a sphere centered about its focus. Consider a fixed point on the axis, well removed from the focus, and the two spherical wave fronts passing through this point (see fig. 7). We ask the question, how does the distance between these spheres behave as a function of aperture?

If $x$ and $y$ are the coordinates of a point on the

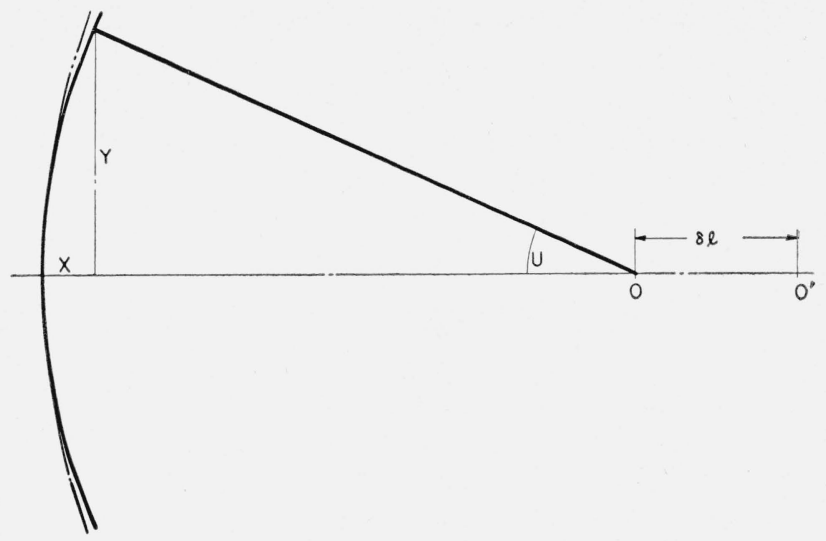

Figure 7.

Two wave fronts in light of different colors in the neighborhood of the axis. The longitudinal abberration is $\delta l$. The wave front separation is $\Delta x$

sphere of radius $r$, then $x=\frac{1}{2} y^{2} r^{-1}$ is valid for a meridian section in the neighborhood of the axis, where $r$ is the radius. The $x$-axis coincides with the optical axis, and $x=0$ at the vertex of the sphere. Differentiating with respect to $r$, one has $\Delta x=-\frac{1}{2}$ $y^{2} r^{-2} \Delta r$ as the approximate displacement of the sphere of radius $r+\Delta r$ from the sphere of radius $r$. But $\Delta x=-\Delta V / N^{\prime}$, where $\Delta V=\Sigma D \Delta N$ and $N^{\prime}$ is the final index (see eq (3), section 2). Then $\Delta V=\frac{1}{2} N^{\prime}$ $y^{2} r^{-2} \delta l$, since $\delta l=\Delta r$. Also sin $U=y r^{-1}$, and so

$$
\Delta V=\frac{1}{2} N^{\prime} \delta l \sin ^{2} U \text {. }
$$

Equation (5) shows that the curve of $\Delta V$ against $\sin U$ is a circle or parabola for small values of $U$ and that the curvature is $N^{\prime} \delta l$. This then is the paraxial correspondence desired. Another way of expressing this result is to consider $\Delta V$ as a power series in $\sin U$. For an axial object point this series has the form $\Delta V=c_{0}+c_{2} \sin ^{2} U+c_{4} \sin ^{4} U+\ldots$ The odd powers of sin $U$ are absent because of symmetry. The first term $c_{0}$ is of no importance because it only represents a displacement of the reference point, which indicates that the wave fronts for the two wavelengths do not pass through the same axial point. This term $c_{0}$ is similar to the quantity $\Sigma d \Delta N$, which it is customary to subtract from $\Delta V$.

The coefficient $c_{2}$ depends upon the paraxial difference in focus, and by eq (5) $c_{2}=\frac{1}{2} N^{\prime} \delta l$. The coefficients $c_{4}, c_{6}$, etc. are related to the chromatic difference in spherical aberration. It might be emphasized that the $D-d$ method gives the sum of this series precisely for small wavelength variations and approximately for finite differences in wavelength.

In practice, the designer determines $\delta l$ either exactly from a paraxial ray trace, or approximately by a formula given below. Then $\Delta V$ is determined from the $D-d$ method for a number of points throughout the aperture. (Frequently the values at 
the margin and the 0.7 zone are sufficient.) Then a curve can be drawn for $\Delta V$ versus $\sin U$ by making use of the value of $\delta l$ to draw the curve near the origin. The curves shown in figure 2 were obtained in this manner.

The approximate formula for $\delta l$ is

where

$$
\delta l=a\left(N^{\prime} u^{2}\right)^{-1}
$$

$$
a=\sum_{k} y_{k} i_{k}\left(\Delta N_{k}-\mu_{k} \Delta N_{k}^{\prime}\right) \text {. }
$$

The meanings of the above symbols, which all refer to the paraxial ray, are as follows:

$u$ is the paraxial inclination angle in the final medium.

$y$ is the height of incidence.

$\mu=N / N^{\prime}$ at the refractive surface.
$\Delta N$ and $\Delta N^{\prime}$ are the differences in indices for two wavelengths for the media preceding and following the refracting surface.

The subscript $k$ refers to the $k$ th surface and the sum runs over oll the refracting surfaces in the system, the expression $\left(N^{\prime} u^{2}\right)^{-1}$ refers to the final medium.

Combining eq (6) with eq (5), one finds that $\Delta V=\frac{1}{2} a$ if one takes $\sin U=u$. This means that if one traces a paraxial ray through the system and computes $a$ by eq (6), then $\Delta V$ along this ray is simply $\frac{1}{2} a$.

WAshington, July 15, 1953. 